\title{
Radioimmunotherapy-time to be upfront and fractionated?
}

For patients with follicular lymphoma, a number of therapeutic options are available, many of which rely on rituximab-based chemoimmunotherapy in the first line. For patients with relapsed disease, two radioimmunotherapies have been FDA approved, one of which is approved in Europe- ${ }^{90} \mathrm{Y}$-ibritumomab tiuxetan. Data from the past few years support the use of radioimmunotherapy in the first-line setting. Now, Tim Illidge and colleagues report phase II data showing that ${ }^{90} \mathrm{Y}$-ibritumomab tiuxetan is effective upfront in a fractionated regimen in patients with advanced-stage follicular lymphoma.

"Radioimmunotherapy has proven to be highly active (with high rates of durable remissions) in relapsed follicular lymphoma," says Illidge. "Thus, we wanted to investigate using radioimmunotherapy alone as front-line therapy in patients with large tumour burdens." The researchers enrolled 74 patients, $78 \%$ of whom had stage III-IV disease and $44 \%$ of whom were considered high risk according to the Follicular Lymphoma International Prognostic Index. Overall, 32 patients had bone marrow infiltration, for which this was $>20 \%$ for 13 patients - these patients in particular are not generally offered radioimmunotherapy. "We also wanted to test whether using 4-weekly infusions of rituximab would clear the bone marrow infiltration and permit a broader population of patients to benefit from treatment," explains Illidge.

\section{1 ...patients ... could be pretreated with rituximab and then go on to receive the radioimmunotherapy 77}

Given that antibodies are large molecules, a single dose might not distribute homogeneously in patients with bulky disease. Illidge and his team examined a fractionated regimen, hypothesizing that this approach would also enable dose escalation and potentially improve response rates and survival.
Indeed, the overall response rate was 94.4\% and complete response rate was $69.4 \%$. The 3 -year progression-free survival rate after a median follow-up of 3.1 years was estimated to be $58 \%$; overall survival was $95 \%$ at 3 years. "Furthermore, patients with heavy bone infiltration could be pretreated with rituximab and then go on to receive the radioimmunotherapy."

Importantly, ${ }^{90} \mathrm{Y}$-ibritumomab tiuxetan radioimmunotherapy was well tolerated, with grade 3 or 4 infections occurring in $<3 \%$ of the cohort. "We would like to test this approach against the standard multiple cycles of rituximab-based therapy to evaluate whether ${ }^{90} \mathrm{Y}$-ibritumomab tiuxetan is as effective and preferred by patients," concludes Illidge.

Mina Razzak

Original article Illidge, T. M. et al. Fractionated ${ }^{90} \mathrm{Y}$-ibritumomab tiuxetan radioimmunotherapy as an initial therapy of follicular lymphoma: an international phase II study in patients requiring treatment according to GELF/BNLI criteria. J. Clin. Oncol. doi:10.1200/ JC0.2013.50.3110 